\title{
Delayed hemiparkinsonism after closed head injury
}

\section{Hemiparkinsonismo tardio após traumatismo craniano fechado}

Monalisa da Silveira Dias ${ }^{1}$, Pedro Renato Brandão ${ }^{1,2}$, Talyta Grippe ${ }^{1,3}$, Cassio Jovem ${ }^{4,5}$, Marcelo Gomes ${ }^{6}$, Flávio Faria Pereira ${ }^{1}$

A 40-year-old man complained of insidiously-reduced right arm dexterity, which started three years previously. He had had a severe closed head injury 19 years before. At the examination, he presented with rigidity, akinesia, and dystonia uniquely over the right side. According to Crouzon and Justin-Besancon, the criteria for traumatic secondary parkinsonism are severe trauma, brain concussion, and a temporal relationship between the trauma and symptoms ${ }^{1}$. In this patient, presynaptic dopaminergic imaging corroborated nigrostriatal denervation induced, presumably, by a previous traumatic hemorrhage. As a result, neuroimaging (Figures 1 and 2) showed specific features that validated the diagnosis of parkinsonism secondary to a traumatic etiology $y^{2,3}$.
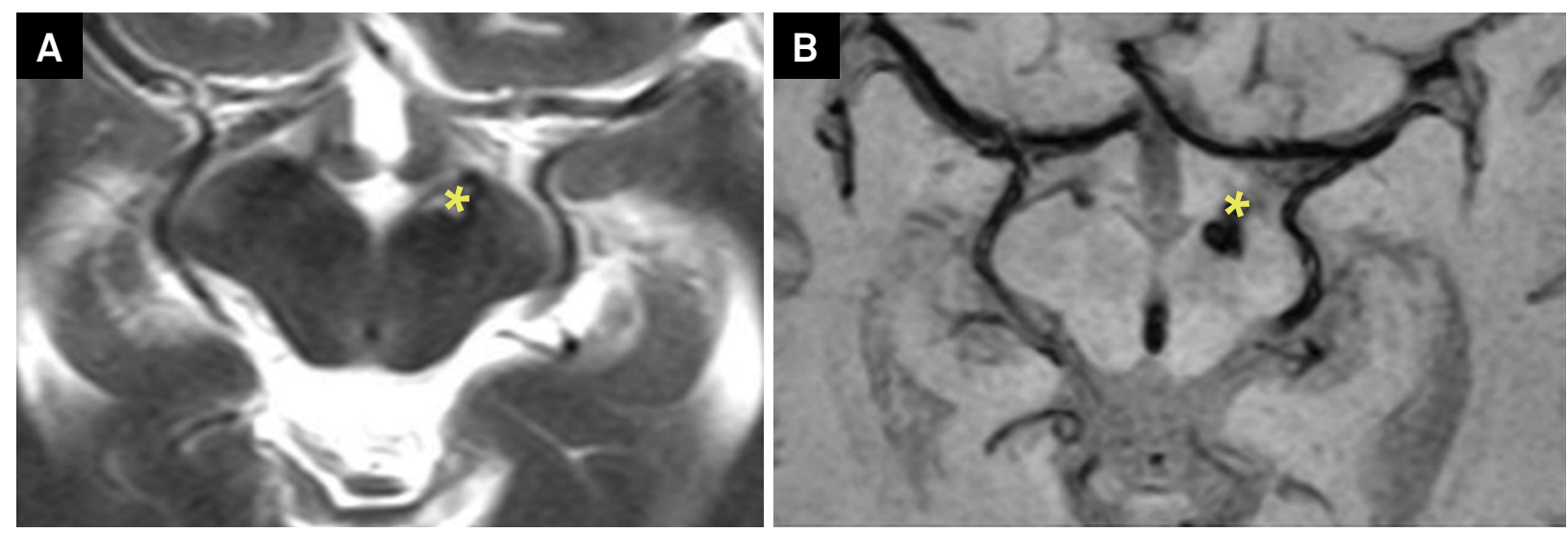

Figure 1. Magnetic resonance imaging, with T2 (A), and susceptibility weighted imaging (B), discloses, in detail, a focal lesion with hemosiderin deposits, over the left cerebral peduncle and substantia nigra.

\footnotetext{
${ }^{1}$ Hospital de Base do Distrito Federal, Unidade de Neurologia, Centro de Distúrbios do Movimento e Doença de Parkinson, Brasília DF, Brasil; ${ }^{2}$ Congresso Nacional, Câmara dos Deputados, Departamento Médico, Brasília DF, Brasil;

${ }^{3}$ Universidade de Brasília, Faculdade de Medicina, Programa de Pós Graduação, Brasília DF, Brasil;

${ }^{4}$ Clínica Villa Rica, Brasília DF, Brasil;

${ }^{5}$ Hospital de Base do Distrito Federal, Departamento de Radiologia, Brasília DF, Brasil;

${ }^{6}$ Núcleos Medicina Nuclear, Brasília DF, Brasil.
}

Correspondence: Talyta Cortez Grippe; Faculdade de Medicina da Universidade de Brasília; Campus Universitário Darcy Ribeiro, Asa Norte; $70904-970$ Brasília DF, Brasil; E-mail: talytagrippe@gmail.com

Conflict of interest: There is no conflict of interest to declare.

Received 18 November 2016; Received in final form 29 August 2017; Accepted 05 September 2017. 

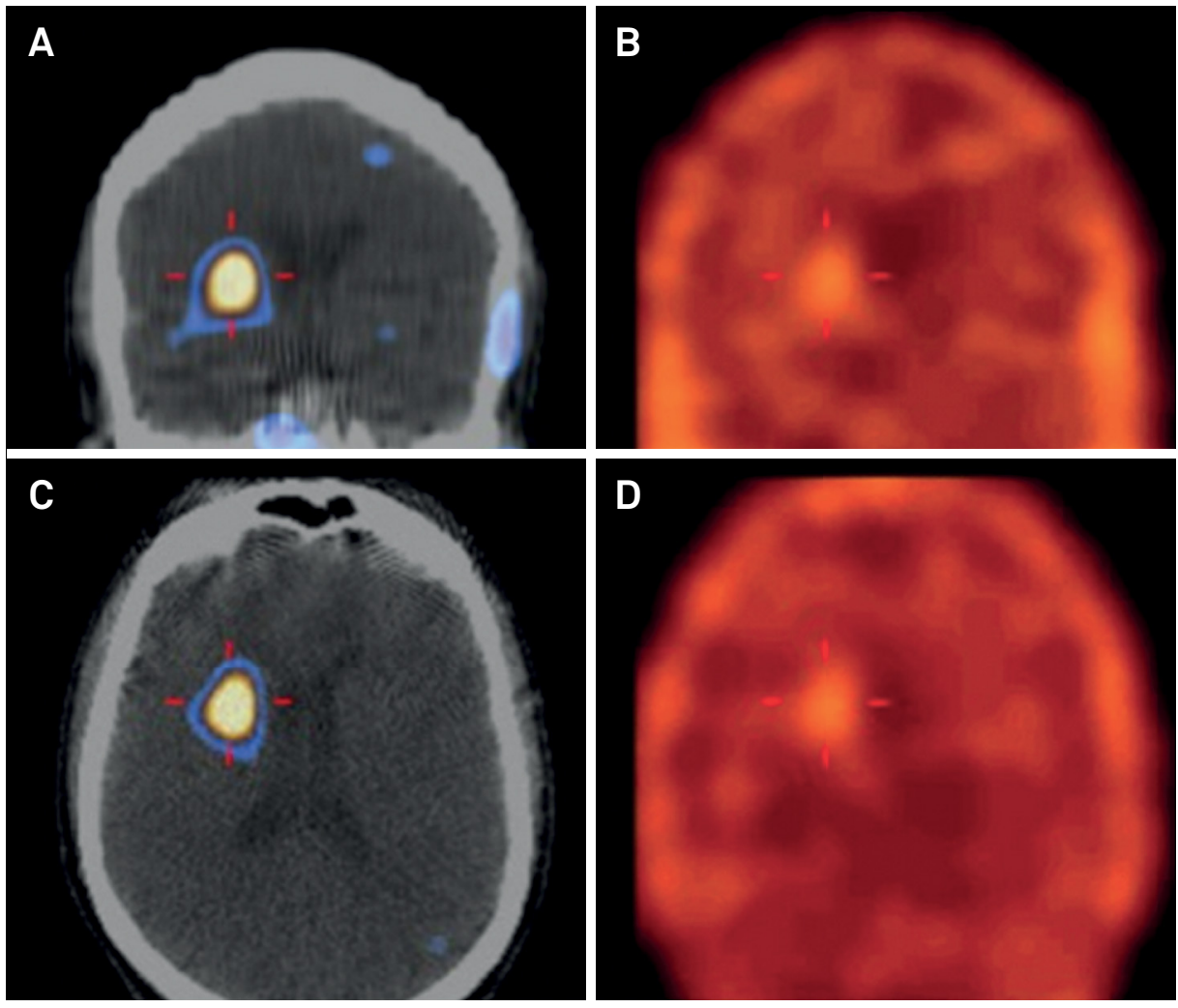

Figure 2. Scintigraphic imaging with single photon emission computed tomography shows normal $99 \mathrm{~m} T \mathrm{~T}-\mathrm{TRODAT}$ uptake in the right striatum, and absence of the radionuclide concentration on the left side.

\section{References}

1. Crouzon O, Justin-Besancon L-E. Post-traumatic parkinsonism. Presse Med. 1929;37:1325-7. https://doi.org/10.1016/j.lpm.2017.01.018

2. Loher TJ, Krauss JK. Dystonia associated with

pontomesencephalic lesions. Mov Disord. 2009;24(2):157-67.

https://doi.org/10.1002/mds.22196
Krauss JK, Jankovic J. Head injury and posttraumatic movement disorders. Neurosurgery. 2002;50(5):927-39. https://doi.org/10.1097/00006123-200205000-00003 